\title{
Integrating printed microfluidics with silicon photomultipliers for miniaturised and highly sensitive ATP bioluminescence detection
}

M. F. Santangelo, S. Libertino, Anthony Turner, Daniel Filippini and Wing Cheung Mak

The self-archived postprint version of this journal article is available at Linköping University Institutional Repository (DiVA):

http:// urn.kb.se/ resolve?urn=urn:nbn:se:liu:diva-142824

N.B.: When citing this work, cite the original publication.

Santangelo, M. F., Libertino, S., Turner, A., Filippini, D., Mak, W. C., (2018), Integrating printed microfluidics with silicon photomultipliers for miniaturised and highly sensitive ATP bioluminescence detection, Biosensors \& bioelectronics, 99, 464-470. https:// doi.org/ 10.1016/j.bios.2017.07.055

Original publication available at:

https:// doi.org/ 10.1016/j.bios.2017.07.055

Copyright: Elsevier

http:// www.elsevier.com/

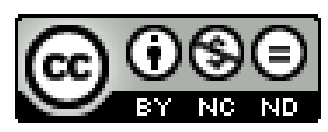




\title{
Integrating printed microfluidics with silicon photomultipliers for miniaturised and highly sensitive ATP bioluminescence detection
}

\author{
M.F. Santangelo ${ }^{\mathrm{a}}$, S. Libertino ${ }^{\mathrm{a}}$, A.P.F. Turner ${ }^{\mathrm{b}}$, D. Filippinic ${ }^{\mathrm{c}}$ and W.C. Mak ${ }^{\mathrm{b}, *}$ \\ ${ }^{a}$ CNR IMM, VIII strada Z.I., 5, 95121 Catania, Italy; \\ ${ }^{\mathrm{b}}$ Biosensors and Bioelectronics Centre, Department of Physics, Chemistry and Biology - IFM, Linköping University, S- \\ 58183 Linköping, Sweden;

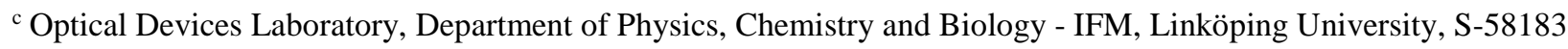 \\ Linköping, Sweden; \\ *E-mail: wing.cheung.mak@liu.se; Tel.: +46-0-1328-6921.
}

\begin{abstract}
Bioluminescence has been widely used for important biosensing applications such as the measurement of adenosine triphosphate (ATP), the energy unit in biological systems and an indicator of vital processes. The current technology for detection is mainly based on large equipment such as readers and imaging systems, which require intensive and time-consuming procedures. A miniaturised bioluminescence sensing system, which would allow sensitive and continuous monitoring of ATP, with an integrated and low-cost disposable microfluidic chamber for handling of biological samples, is highly desirable. Here, we report the design, fabrication and testing of 3D printed microfluidics chips coupled with silicon photomultipliers (SiPMs) for high sensitive real-time ATP detection. The 3D microfluidic chip reduces reactant consumption and facilitates solution delivery close to the SiPM to increase the detection efficiency. Our system detects ATP with a limit of detection (LoD) of $8 \mathrm{nM}$ and an analytical dynamic range between 15 $\mathrm{nM}$ and $1 \mu \mathrm{M}$, showing a stability error of $3 \%$, and a reproducibility error below of $20 \%$. We demonstrate the dynamic monitoring of ATP in a continuous-flow system exhibiting a fast response time, $\sim 4 \mathrm{~s}$, and a full recovery to the baseline level within 17s. Moreover, the SiPM-based bioluminescence sensing system shows a similar analytical dynamic range for ATP detection to that of a full-size PerkinElmer laboratory luminescence reader.
\end{abstract}

\section{Keywords}

ATP bioluminescence detection; Silicon Photomultiplier; Disposable 3D Lab-on-chip; Continuous flow monitoring; Miniaturised sensing system. 


\section{Introduction}

Commercially available platforms to perform biological testing or environment monitoring are expensive, bulky, characterised by high power consumption, and require substantial amounts of reactants. The growing need for decentralised monitoring (e.g. of work or natural environments) is driving the scientific community to develop miniaturised platforms to facilitate the production of inexpensive and easy-to-use systems. Examples where microbial content or cells need to be measured include clinical analysis, determination of bacterial pollution levels (Tanii et al., 2001), detection of contamination of drinking water, food safety and public hygiene (Champiat et al., 2001). Living organisms can be detected by a variety of methods, but ATP-driven bioluminescence is both sensitive and, unlike the use of fluorescent labels, does not require external sources of light excitation. Bioluminescence detection of living cells (Stanley, 1989; Steinberg et al., 1995; Gracias and McKillip, 2004) exploits the reaction of ATP with luciferin catalysed by the enzyme luciferase, to yield an intermediate complex (luciferyl adenylate) that combines with oxygen to produce oxyluciferin and a photon at $560 \mathrm{~nm}$. The full reaction is (Tanii et al., 2001):

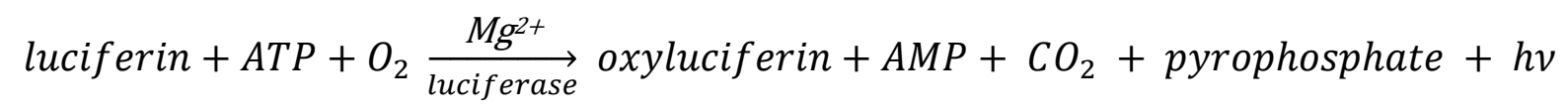

Luciferase systems are used in biomedical research, such as for in vivo studies of infections with bioluminescent pathogens (Xiong et al., 2005), cancer progression using bioluminescent cancer cell lines and reconstitution kinetics using bioluminescent stem cells (Di Rocco et al., 2008). ATP bioluminescent assays are a reliable technique to measure the number of viable cells (Brolin and Wettermark, 1992; Ahn et al., 2006) or biomolecules (Min, 2007) in/on a sample and are widely used to indicate microbial contamination.

The light emitted can be detected using a silicon photomultiplier (SiPM), which is a solid-state photodetector consisting of a pixelated array in which each pixel is composed of a series of a quenching resistors and a single photon avalanche diode (SPAD), operating in Geiger mode (Sciacca et al., 2003; Mazzillo et al., 2008; 2009; Pagano et al., 2013). The presence of the quenching resistor (some hundreds of $\mathrm{k} \Omega$ ) for each SPAD allows the single pixel to work as an independent photon counter, since it turns off the avalanche triggered by the generation of electronhole (e-h) pairs inside the SPAD (digital operating mode). All pixels are connected in parallel to a single common resistor (some tens of $\Omega$ ) where the output signal is collected. The current produced is proportional to the number of fired pixels, since the amount of charge produced in each pixel during the avalanche event is the same (analog operating mode). The output signal is a current level that can be easy manipulated and visualised on-line. Several works (Michalet et al., 2014; 
Santangelo et al., 2016; Burri et al., 2014; Santangelo et al., 2015; Pagano et al., 2016) describe SiPMs as fluorescence detectors for biosensing thanks to their high quantum efficiency, high gain, high speed, low operating voltage, simple output electronics, and single photon sensitivity.

The reaction chamber in a bioluminescence detection system must be designed to obtain the maximum signal while to minimising reagent consumption. Fluidic chips commonly used for biological assays in most laboratories are fabricated using traditional photolithographic methods resulting in polydimethylsiloxane (PDMS) on glass lab-on-chip (LOC) devices. Fast prototyping using $3 \mathrm{D}$ printers is emerging as a versatile tool to directly produce a physical object from a computer aided design $\left(\mathrm{CAD}^{\circledR}\right)$, thus avoiding expensive fabrication facilities and the steep learning curve required for effective microfabrication. In this work, we used the unibody-LOC (ULOC) 3D printing concept developed for LOC (Comina et al., 2014; 2015) and optical devices (Comina et al., 2015; 2016). ULOC utilises consumer grade stereolithography (SLA) 3D printers to produce a single monolithic printout incorporating all the features required to connect and execute the LOC functions. In ULOC design, all fluidic channels are created open on one side, thus securing the effective removal of uncured resin and robust geometric consistency even for long channels. The good SLA surface roughness then allows the open sided channels to be sealed with adhesive tape in one single operation that simplifies the manufacturing and minimises the development costs. The resulting LOC is robust, reproducible (fabricated by the printer) and, as any microfluidic configuration, it allows reduced reactant consumption and results in compact and sensitive systems. Such an approach enables potentially low-cost bioluminescent detection systems to be developed with wide-ranging applications in chemical and biological analysis and clinical diagnostics.

We designed and fabricated a disposable lab-on-chip for luciferase bioluminescence detection (with ATP detection as the model). The 3D microfluidic chip interface allowed the sample solution to be delivered close to the SiPM to increase the detection efficiency of the bioluminescence light emitted from the sample solution. The analytical performance of the low-cost and miniaturised SiPM-based ATP detection system developed was compared with a bench-scale commercial bioluminescent reader. We demonstrate that the two systems exhibit comparable sensitivity and analytical window for the detection of low levels of ATP.

\section{Materials and methods}

\subsection{Standard preparation and ATP bioluminescence measurements}

The ATP bioluminescence assays used in this work (ATP Determination Kit (A22066) from Molecular Probes, 2005) offers a convenient bioluminescence assay for quantitative determination of ATP with recombinant firefly luciferase and its substrate D-luciferin. It is based on the reaction 
reported in eq. (1). Light emission occurs at $\sim 560 \mathrm{~nm}$ at $\mathrm{pH} 7.8$. The assay involves the lightemitting pigment luciferin and the enzyme luciferase to catalyse luciferin oxidation. As stated in eq. (1), the reaction requires magnesium ions $\left(\mathrm{Mg}^{2+}\right)$ as a cofactor. The amount of emitted light is directly associated with the ATP concentration. Carbon dioxide $\left(\mathrm{CO}_{2}\right)$, adenosine monophosphate (AMP) and pyrophosphate are released as by products.

Molecular Probes' ATP Determination Kit provided the stock ATP solution, at a concentration of 5 mM. We prepared seven different concentrations through dilution: $1 \mu \mathrm{M} ; 500 \mathrm{nM} ; 250 \mathrm{nM} ; 25$ nM; 62.5 nM; 31.25 nM; 15.625 nM. The “Standard Reaction Solution (SRS)” was prepared using: $\mathrm{dH}_{2} \mathrm{O}$ (deionised water), 20X Reaction Buffer, dithiothreitol (DTT) to a concentration of 0.1 M, Dluciferin to a concentration of $10 \mathrm{mM}$ and firefly luciferase $(5 \mathrm{mg} / \mathrm{ml})$. Mixing the SRS and ATP produces bioluminescence at $560 \mathrm{~nm}$. The ATP concentrations were tested in continuous flow mode by simply changing the flow rate (FR) ratio of ATP and SRS. The FR of SRS was fixed at 19.2 $\mathrm{ml} / \mathrm{h}$, while the FR of ATP solution with a concentration of $1 \mu \mathrm{M}$ ranged from 0.6 to $19.2 \mathrm{ml} / \mathrm{h}$, in order to obtain a final ATP concentration in the range $15.625-500 \mathrm{nM}$, respectively.

\subsection{Preparation of cell lysate sample and real sample measurements}

Escherichia coli (E. coli) O157:H7 ATCC 43889 (gram negative) were grown in brain heart infusion (BHI) broth at $37^{\circ} \mathrm{C}$ for 18 hours. The $E$-coli was harvest by centrifuge (5,000 gram for 10 minutes), followed by 2 times washing with phosphate buffer saline (PBS) and disperse with PBS to achieve a cell density of $10^{8} \mathrm{CFU} / \mathrm{ml}$. Then, $1 \mathrm{ml}$ of cell lysis buffer (B-PER ${ }^{\mathrm{TM}}$, ThermoFisher, USA) composed of phosphate buffer saline (PBS), lysoyme, triton x-100 and EDTA was added to 1 $\mathrm{ml}$ of the $E$. coli suspension and incubate for 30 minutes with gentle mixing. The cell lysate was then centrifuged at 16,000 gram for 20 minutes to remove the insoluble fraction, and the cell lysate supernatant was collected. The intracellular ATP concentration of the E-coli cell lysate was measured with standard addition method (Romanova et al., 2003). The measurement of ATP in cell lysate is performed with similar procedure described in section 2.1.

\subsection{Standard optical characterisation}

The ATP determination kit solutions prepared were tested using a PerkinElmer Victor 2030 as a "gold standard". The solutions were spotted on a 96 well standard plate, to volumes of $100 \mu \mathrm{l}$ in each well. The wells were filled with the seven solutions in triplicate. A reference triplicate was also spotted using $\mathrm{dH}_{2} \mathrm{O}$. The plate was then inserted in the instrument with a delay time of few seconds with respect to the reaction start. The instrument recorded the signals from each well sequentially. A scan of the full plate (only the filled wells, i.e. 24 wells) required few minutes. A proprietary 
piece of software provided the luminescence signal intensity in counts per second. The values reported in the paper are the averages of the three signals recorded for each ATP concentration after subtraction of the reference signal.

\subsection{SiPM details}

A Silicon Photomultiplier, developed by the R\&D Sensor Team at STMicroelectronics in Catania (Mazzillo et al., 2008; 2009) was used in this work. It comprises 25 pixels, where every pixel is a Single Photon Avalanche Diode (SPAD) operating in Geiger mode (Sciacca et al., 2003). Each pixel is insulated from the others optically and electrically thanks to the presence of excavations fabricated all around them, and filled by oxide or metal (optical trenches) (Pagano et al., 2011). The SiPM used for this application exhibits a physical size of $0.4 \times 0.4 \mathrm{~mm}^{2}$ (Fig. 1a), a Dark Count (DC) of $7.9 \cdot 10^{4} \mathrm{~Hz}$, a cross-talk probability of 0.03 (Santangelo et al., 2016; Pagano et al., 2012; Finocchiaro et al., 2009) and a cost for manufacturer below $1 €$. Moreover, we have previously shown that it works at low operating voltage (-30V), with high speed ( 150ps), high gain $\left(\sim 1.5 \cdot 10^{6}\right)$, simple output electronics and high sensitivity ( 76 $\left.\pm 2 \mathrm{nA}\right)$ (Santangelo et al., 2016).

\subsection{Setup description}

Two arrangements of the experimental setup were developed by using two different sample holders: glass cuvettes and 3D printed fluidic chips (Fig. 1b, Fig. 1c and Fig. 1d, respectively). In both arrangements, the sensor (SiPM) is placed inside an appropriately designed box (MiniDom) (Libertino et al., 2012) and biased through a 4200 Keithley Source Meter that acquires the SiPM output signal. The MiniDom has an opportunely fabricated hole $(\mathrm{d}=\sim 6 \mathrm{~mm})$ in the upper shield. A glass tube $(\mathrm{d}=\sim 5 \mathrm{~mm})$, or the $3 \mathrm{D}$ reaction chamber $(\mathrm{d}=\sim 6 \mathrm{~mm})$, containing the sample to be analysed was inserted in the hole. In both cases, the SiPM active area was very close to the reaction region (approximately $2 \mathrm{~mm}$ ). All the system components were placed inside a dark box to reduce the optical background noise. In the 3D chip configuration, the continuous monitoring of the reaction flow was performed using two NE-1010 Higher Pressure Programmable Single Syringe Pumps to inject the ATP solutions and SRS (http://www.syringepump.com/NE-1000.php\#NE-1010). 

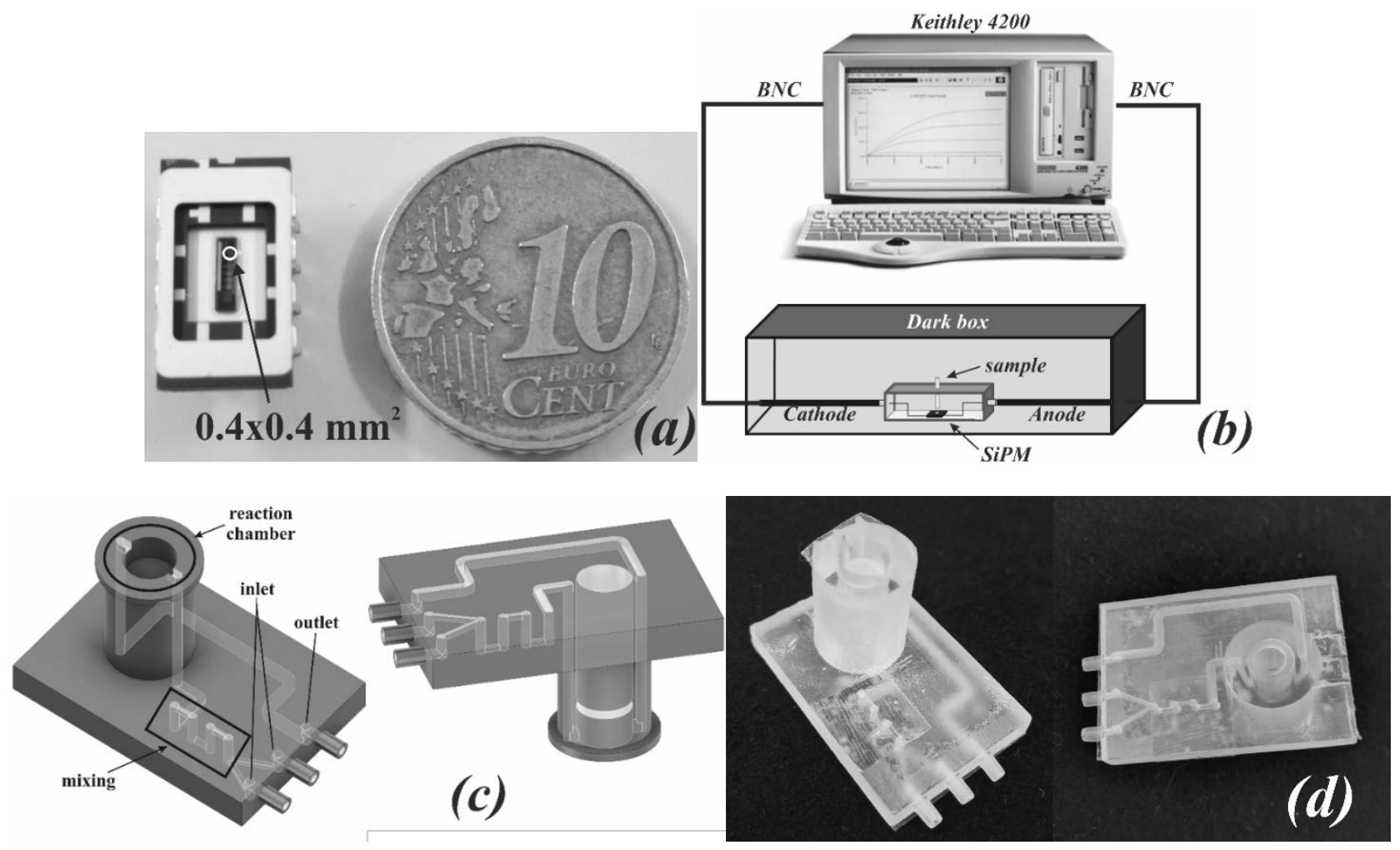

Fig. 1: (a) Picture of 25 pixels SiPM; (b) schematic of experimental setup based on SiPM technology; (c) 3D printed microfluidic chip; (d) picture of 3D printed microfluidic chip.

The measurements were performed by biasing the SiPM with a Keithley 4200, using a set-up which allowed control of the bias voltage (fixed at $-30 \mathrm{~V}$ ) and the measurement conditions (time of measurement), and acquired the output signal automatically. The signal was elaborated offline by a routine developed in Matlab ${ }^{\circledR}$ and Origin ${ }^{\circledR}$. The values and the errors in the experimental points reported were calculated from the signal acquired over the time range 3-25 s (see below) on three different measurements.

\subsection{3 printed chip}

The fluidic chip was designed using CAD software (Autodesk Inventor Fusion ${ }^{\circledR}$, Autodesk Inc.). The reaction chamber and channels were open, providing easy access for functionalisation, and the sub-micro-metric surface finishing enabled sealing with regular adhesive tape (Comina et al., 2014; Comina et al., 2016). A Form 1+ (http://formlabs.com/3d-printers/form-1-plus/) 3D printer was used to fabricate the microfluidic chip, using the proprietary resin Clear Type 02. The exact composition of the resin is not disclosed by the manufacturer, but includes different proportions of modified acrylate and acrylate oligomer, epoxy monomer, acrylate monomer, photo initiator and additives as the principal components (Comina et al., 2014). Less than 30 minutes were needed to print the chip and the prototype cost is $0.65 €$. After printing, the chip was sonicated in ethanol for a few minutes and finished with regular adhesive tape to close the open side of the channels and the bottom of the reaction chamber. In addition to the simplicity, cost effectiveness and short 
development time, it is worth noting that the unusual geometry required for this detection would have demanded an especially expensive and cumbersome classical manufacturing process. The microfluidic chip comprised two inlet ports with an internal radius of $500 \mu \mathrm{m}$, through which ATP and standard reaction solution were separately injected. The ports are part of the ULOC and were designed with and external diameter sufficient to allow a tight insertion of $1 \mathrm{~mm}$ inner diameter silicone tubing. The free side of the tubing is then used to attach regular Teflon tubing to the offchip instrumentation. In this way, the need for expensive connectors and leakage problems are entirely avoided. Solutions are directed through a 3D bulk serpentine mixing feature (Comina et al., 2016), using NE-1010 Higher Pressure Programmable Single Syringe Pumps (http://www.syringepump.com/NE-1000.php\#NE-1010) and circulate to the $100 \mu \mathrm{l}$ measuring chamber immediately next to the detector (Fig. 1c). A returning channel connected to a beaker and opened to the external environment serves as waste reservoir.

\section{Results and discussion}

The static and dynamic performance of the SiPM for ATP bioluminescence detection were evaluated by measuring different concentrations of ATP, ranging from $15 \mathrm{nM}$ to $1 \mu \mathrm{M}$, and comparing the two experimental setups described in Section II.

\subsection{Static performance}

Static performance of the optical system was carried out using the SiPM and glass cuvette setup. The experimental data obtained are reported in Fig. 2. Signals corresponding to water and system dark current were also measured as controls.
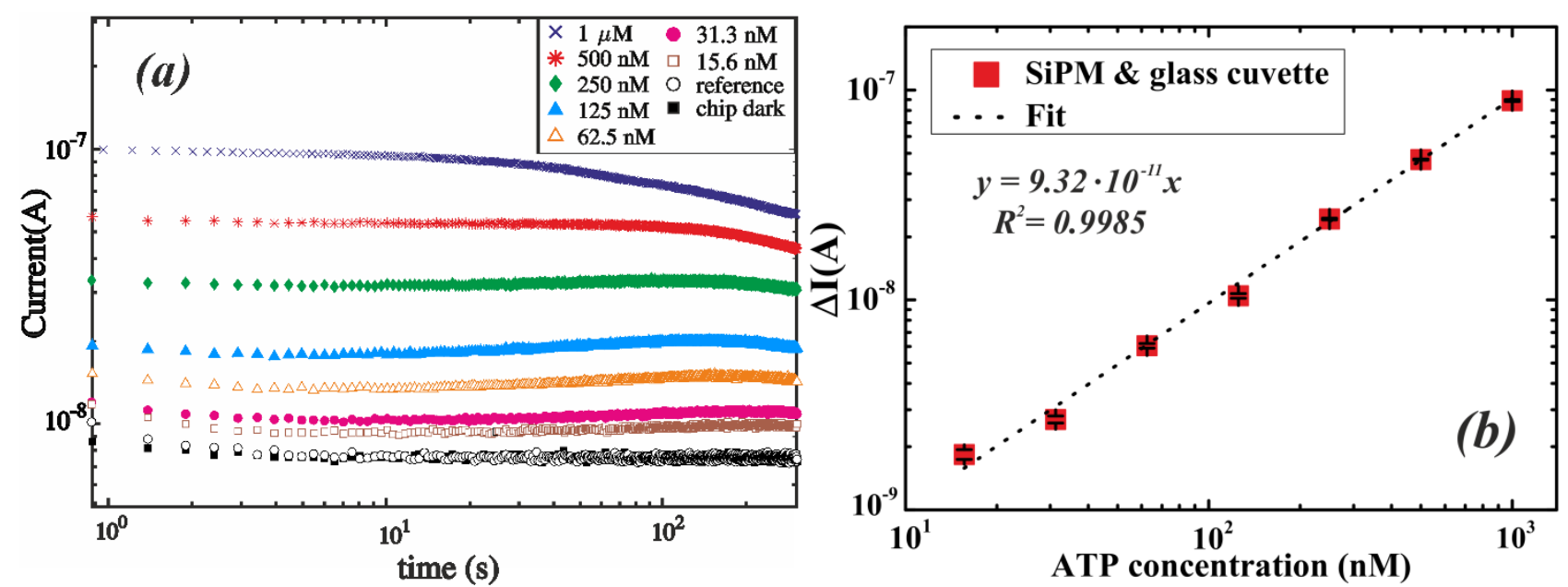

Fig. 2: (a) ATP bioluminescence signal versus time for ATP concentration of: 1uM (blue cross points); 500nM (red asterisk points); $250 \mathrm{nM}$ (green diamond points); $125 \mathrm{nM}$ (light blue filled triangles); 62.5nM (orange empty triangles); $31.25 \mathrm{nM}$ (magenta filled circles); $15.625 \mathrm{nM}$ (brown empty squares). Reference (empty circles) and dark (filled squares) measurements are reported for comparison. (b) Calibration curve of SiPM with glass cuvette system. 
Figure 2 illustrates the good performance of the SiPM for monitoring ATP bioluminescence. It was able to detect the weak bioluminescence signals emitted by very low ATP concentrations (15.6 nM) with a signal-to-noise-ratio (SNR) of $2 \mathrm{~dB}$. It delivered a sensitivity of $9.32 \cdot 10^{-2} \mathrm{~A} / \mathrm{M}$ and a limit of detection (LoD) of $3.7 \mathrm{nM}$, defined as the ratio between three times the standard deviation (SD) of zero calibrator (reference signal) and the slope of the calibration curve reported in Fig. 2(b) (Armbruster et al., 1994):

$$
L o D=\frac{3 \cdot S D}{\text { slope }}
$$

The bioluminescence reaction kinetics observed in Fig. 2(a) show that with increasing ATP concentration, the current signal increased and the steady state current remained fairly stable except at high ATP concentration $(1 \mu \mathrm{M})$, exhibiting a decay in the steady state current. After $30 \mathrm{~s}$, a fast decay occurs (please note the horizontal scale is logarithmic). This is probably due to the fast consumption of reactants such as luciferin at high ATP concentration; they start to deplete and slow down the reaction kinetics. This was confirmed by the fact that on reducing the ATP concentration, the decay in steady state current occurred after longer reaction times. As an example, for ATP concentration of $500 \mathrm{nM}$ the luminescence decay occurred after more than $100 \mathrm{~s}$.

By analysing the bioluminescence kinetics in Fig. 2 it is possible to distinguish three different phases: (i) a stabilisation due to the technical time needed by the instrument for the auto setting (about 2s); (ii) an intermediate phase during which the signals are roughly constant; and (iii) a final phase in which the signals slowly decrease due to the SRS consumption.

We also verified the system for real sample application by measuring intercellular ATP in lysate of E.coli cells. The results show that the presence of lysate does not affect the performance of the system and the reference signal (Fig. S1). SNR was also calculated for both cases: standard ATP solutions and lysate of E.coli cells. A similar value of SNR of $26.0 \mathrm{~dB}$ and $26.8 \mathrm{~dB}$ were obtained, respectively. The calculated ATP concentration in the lysate of E.coli $\left(10^{8} \mathrm{CFU} / \mathrm{ml}\right)$ was $15.6 \mu \mathrm{M}$.

The SiPM-cuvette arrangement described above allowed continuous monitoring of ATP bioluminescence, but since ATP solutions and SRS were spotted sequentially inside the cuvette, the bioluminescence can be measured only after the last reactant addition and on the "same" solution, in which there is a decay over time. As a result, the user cannot change the ATP solution during measurements, either to refresh it or to change the relative reactant concentrations. Moreover, the data indicates that the best SNR was obtained within the first $30 \mathrm{~s}$ after the reactants mix, as previously observed in literature (Tanii et al., 2001).

\subsection{Dynamic performance}

The limitations of the static system described in the previous section, were overcome by coupling a microfluidic chip to the SiPM. Hence, thanks to the implementation of two programmable syringe 
pumps, several ATP concentrations could be measured in continuous-flow mode by simply changing the flow-rate ratio of the reactants as described in section 2.1. The flow rate of SRS was fixed at $19.2 \mathrm{ml} / \mathrm{h}$, while the flow rate of ATP solution with a concentration of $1 \mu \mathrm{M}$ ranged from 0.6 to $19.2 \mathrm{ml} / \mathrm{h}$, in order to obtain a final concentration in the range 15.6 - $500 \mathrm{nM}$, respectively. The results for the SiPM and 3D printed microfluidic chip coupled system are summarised in Fig. 3(a).
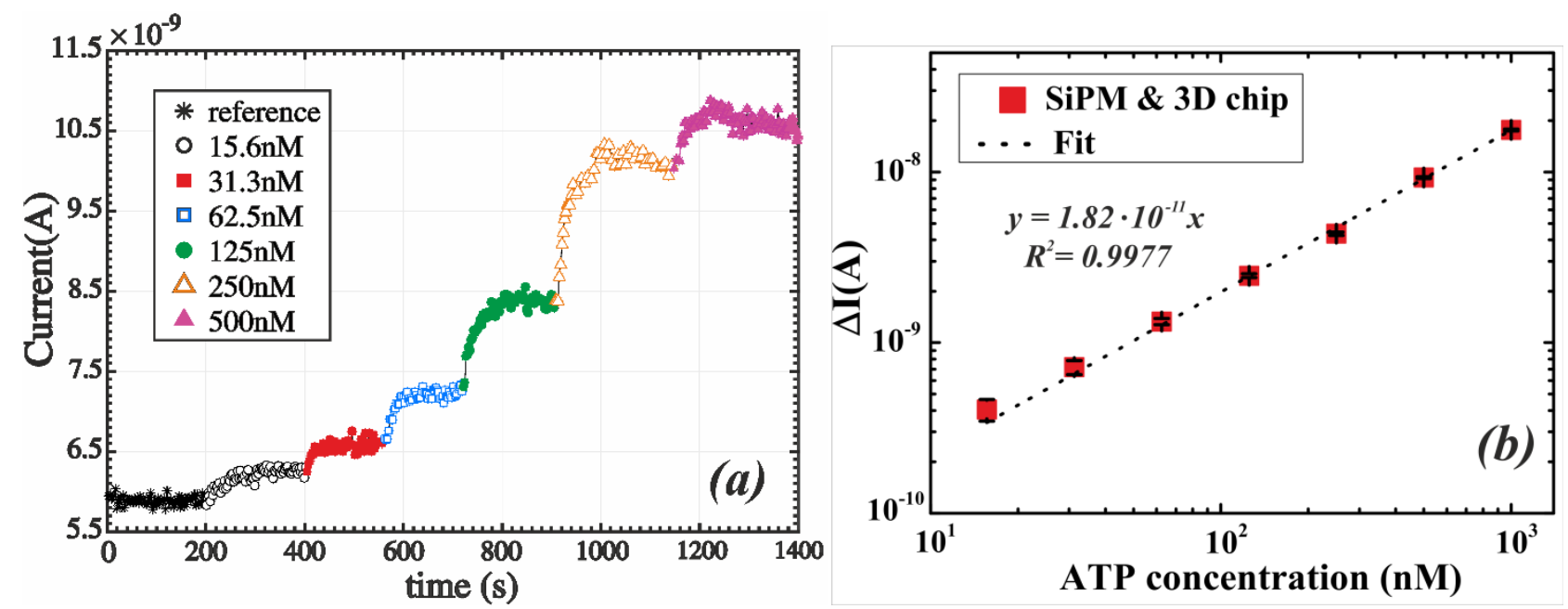

Fig. 3: (a) Continuous flow monitoring of ATP bioluminescence; (b) calibration curve of SiPM with 3D printed microfluidic chip system.

The figure shows that bioluminescence current increases with increasing ATP concentration in the monitored range. The system is very sensitive to ATP concentration changes regulated by the flow rate. It exhibits a sensitivity of $1.82 \cdot 10^{-2} \mathrm{~A} / \mathrm{M}$ and a LoD of $8 \mathrm{nM}$, as extrapolated from the eq. (2) and the calibration curve reported in Fig. 3(b). The LoD calculated in this case is higher than the one obtained for the previous arrangement described above. This difference is due to the different sample holder. In fact, the reaction chamber is characterised by a planar bottom surface, while the cuvette has a round shaped bottom surface, which focuses the light signal on the SiPM active area. As a result, a form of light coupling between the cuvette and the detector is obtained. The comparison of the two system clearly indicate the LoD value is not due to the SiPM sensitivity, but to the optical coupling properties. The optimisation of the chamber surface could significantly improve the coupling between the emitted signal and the active area of the detector, thus further improving both the minimum amount of ATP detected and the LoD itself. In fact, by collecting more signal at low ATP concentrations, the slope of the calibration curve can change thus changing the LoD.

We demonstrated the systems potential for continuous-flow monitoring of bioluminescence by injecting the reactants automatically into the microfluidic chip for detection of low (15.6 nM), medium (125 nM) and high (500 nM) levels of ATP concentration. The SRS was also used as 
buffer solution to wash the channels between sequential repeated measurements. Fig. 4(a) shows that the signal of the time-current response was stable, with a variability error below $3 \%$, during the single repetition and reproducible between repetitions, the error being around $10 \%$ at the highest repetition measured and around $20 \%$ at the lowest. However, the prolonged flow of reactants through microfluidic channels deteriorates the adhesive tape used to close the open side of the channels and the bottom of the reaction chamber. For this reason, disposable use of microfluidic chip is recommended. Moreover, disposable material is preferred for biological samples analysis to avoid cross contamination.

In Fig. 4, the signals were arbitrarily shifted along the y-axis to better visualise the differences between them. For this reason, current was plotted in arbitrary units for each signal. It is known that rise $\left(t_{r}\right)$ and fall $\left(t_{f}\right)$ times are the times needed to switch from $10 \%$ to $90 \%$, or vice versa, of the signal amplitude. Both, the rise and the fall times of the sensor after each injection of ATP solution were analysed. The data clearly show their independence of the ATP concentrations. In fact, all data can be fitted assuming a decreasing exponential equation for both, in the form of:

$$
y=A_{0} \cdot \exp (-t / \tau)+y_{0}
$$

In eq. (3) $A_{0}$ is a constant value depending on the reactant concentrations and whose sign depends on the filling or emptying of the reaction chamber, being positive during chamber emptying and negative during its filling; $\tau$ is the time constant and it has different values during the filling and emptying processes. The filling time constant $\left(\tau_{\mathrm{r}}\right)$ is $1.4 \pm 0.3 \mathrm{~s}$, while the emptying time constant $\left(\tau_{\mathrm{f}}\right)$ is $5.7 \pm 0.2 \mathrm{~s}$ for all repetitions at each concentration, as obtained from data fitting.
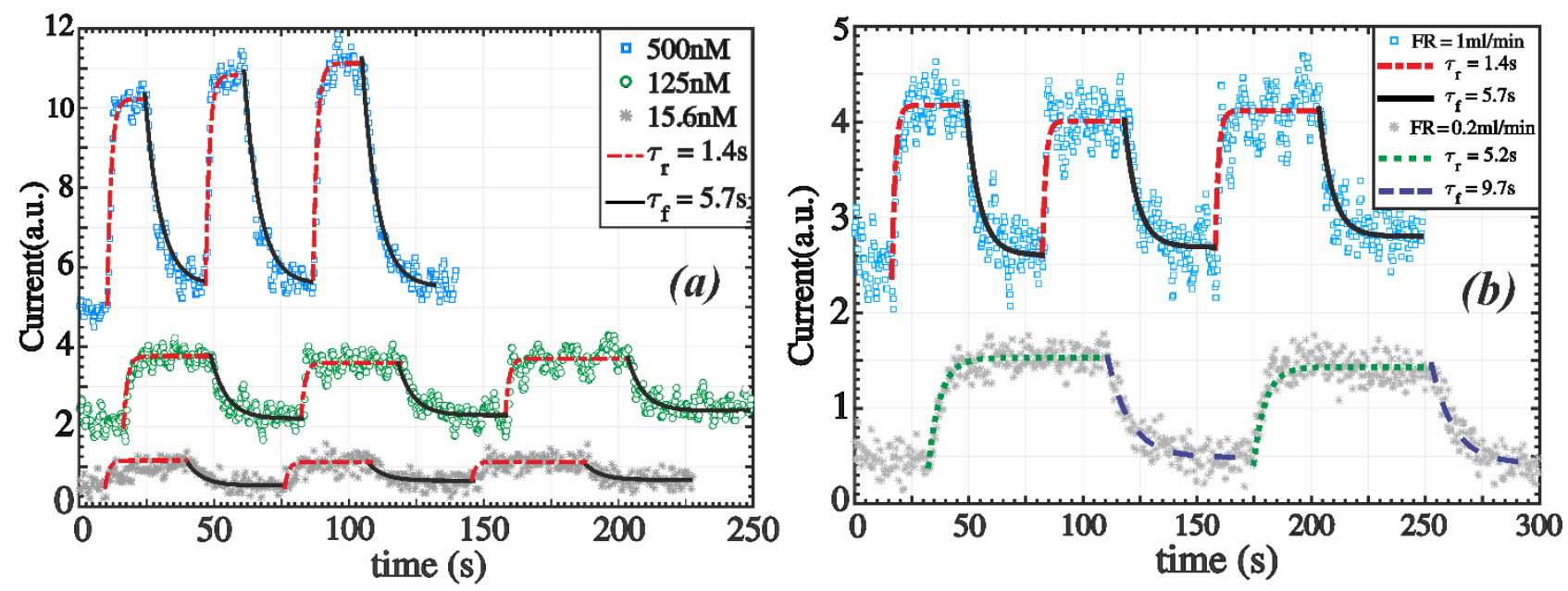

Fig. 4: (a) Bioluminescence signal acquired during a continuous flow monitoring for three different ATP concentrations: 15.6nM (grey star markers); 125nM (green circle markers) and 500nM (light blue square markers); (b) Comparison between signals acquired during continuous flow monitoring of ATP concentrations of $125 \mathrm{nM}$ at two different FR: $1 \mathrm{ml} / \mathrm{min}$ (light blue square markers); 0.2 $\mathrm{ml} / \mathrm{min}$ (grey star data points).

Once the data was fitted, rise and fall time values could be calculated, being each about three times the corresponding characteristic time ( $\tau_{r}$ and $\tau_{f}$, respectively), hence we obtain $\mathrm{t}_{r}=4.2 \pm 0.9 \mathrm{~s}$ and $\mathrm{t}_{f}$ 
$=17.1 \pm 0.6 \mathrm{~s}$. To explain the rise and fall times obtained, the times needed the flow to fill the channels and reaction chamber volumes must be determined. It is known that the ATP assay based on firefly luciferase reaction occurs instantaneously at room temperature (Lundin, 2000), hence it is already taking place when the mixed solution reaches the reaction chamber. The rising time is only due to the chamber filling time. Assuming a flow rate of $1 \mathrm{ml} / \mathrm{min}$, as in Fig. 4(a), a time interval of at least $1.1 \mathrm{~s}$ is needed for the reaction mixture to reach the reaction chamber inlet. To completely fill the chamber, another $\sim 6 \mathrm{~s}$ were needed. As a result, $\sim 7 \mathrm{~s}$ were required for the mixed solution to fill the chamber. Given that the total volume of the mixing region is $\sim 1.45 \cdot 10^{-2} \mathrm{ml}$ (see section 2.5 ), $\sim 0.9 \mathrm{~s}$ are required at the flow rate of $1 \mathrm{~m} / \mathrm{min}$. Summarising, $\sim 2 \mathrm{~s}$ are required for the solution to reach the chamber inlet after the reactants come in contact, and $6 \mathrm{~s}$ to completely fill it. The data in Fig. 4(a) show that the rise time is $\sim 4.2 \mathrm{~s}$, and the time needed to reach $100 \%$ bioluminescence emission (corresponding to $5 \tau_{r}$ ) is $\sim 7 \pm 1.5 \mathrm{~s}$, the same time needed to completely fill the chamber, within experimental error.

The same considerations apply to evaluate the time needed to empty the chamber. To completely clean the microfluidic chip $\sim 28.5 \pm 1$ s (corresponding to $5 \tau_{f}$ ) are required, as experimentally observed.

We assumed that both values, rise and fall time, only depend on the flow rate employed. To verify this assumption, a comparison between the signal obtained for an ATP concentration of $125 \mathrm{nM}$ at two different flow rates, $1 \mathrm{ml} / \mathrm{min}$ and $0.2 \mathrm{ml} / \mathrm{min}$, was performed. The results are summarised in Fig. 4(b). Again, the signals were opportunely shifted along the y-axis to make the differences between them clearer and, for this reason, the current was reported in arbitrary units.

Fig. 4(b) shows as the time constants for the two flow rates are different. In particular, for a flow rate of $0.2 \mathrm{ml} / \mathrm{min}, \tau_{r}=5.2 \pm 0.4 \mathrm{~s}$ and $\tau_{f}=9.7 \pm 0.8 \mathrm{~s}$, respectively for every repetition. The same considerations also apply in this case. Since the flow rate is $0.2 \mathrm{ml} / \mathrm{min}:$ i) $\sim 44 \mathrm{~s}$ are required to ensure the solution reaches and completely fills the reaction chamber; ii) $t_{r}=15.6 \pm 1.2 \mathrm{~s}$; and iii) $48.5 \pm 4 \mathrm{~s}\left(5 \tau_{f}\right)$ are needed to completely clean the microfluidic chip reaction chamber.

\subsection{Commercial platform vs SiPM-based system}

To demonstrate the sensitivity and the improved performance of this system based on SiPM technology, ATP standard solutions with concentration ranging from $15.6 \mathrm{nM}$ to $1 \mu \mathrm{M}$ were measured and compared with a commercial bioluminescence reader. The calibration curve comparison is shown in Fig. 5. The y-axis on the left (in A) refers to SiPM measurements, while the $y$-axis on the right (in counts/s) refers to the commercial reader. The same number of decades were used for both axes to allow an immediate comparison of the data, and the sensitivities, obtained with the different detectors. All the systems compared exhibit similar sensitivities within the experimental errors over the analysed range of ATP concentrations. 


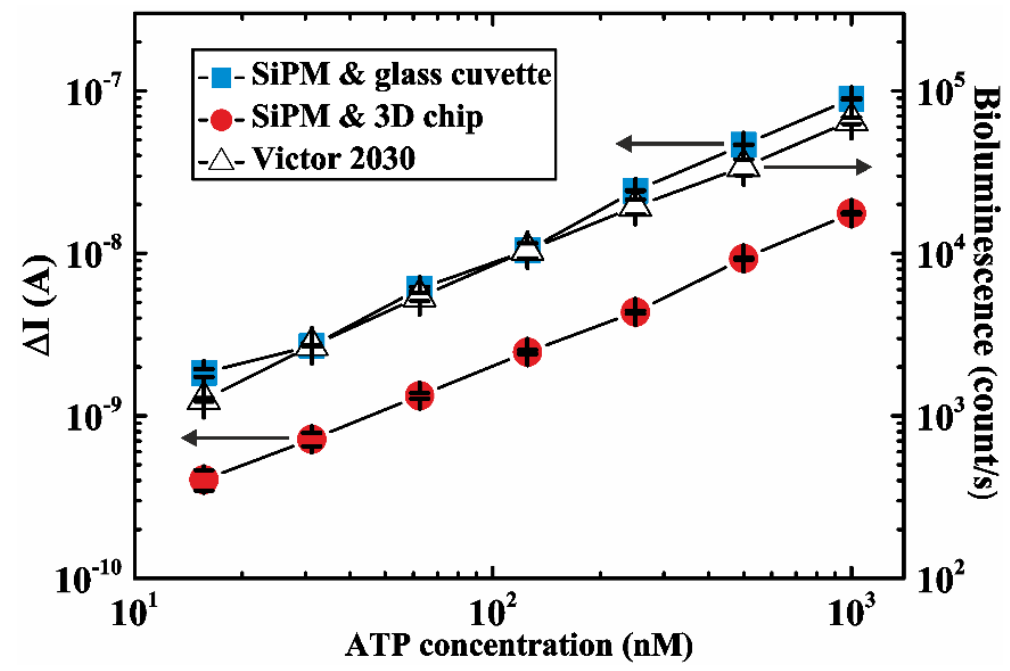

Fig. 5: Comparison between data acquired by SiPM system for glass cuvette (filled squares), SiPM system with 3D chip (filled circles), and commercial reader Victor 2030 (empty triangles).

\begin{tabular}{|l|c|c|c|c|}
\hline \multicolumn{1}{|c|}{ System } & Dynamic range & LoD (nM) & Sensitivity & CV \\
\hline SiPM with cuvette & $>10^{5}$ & 3.7 & $9.32 \cdot 10^{-2} \mathrm{~A} / \mathrm{M}$ & $\sim \% 1$ \\
\hline SiPM with 3D chip & $>10^{5}$ & 8 & $1.82 \cdot 10^{-2} \mathrm{~A} / \mathrm{M}$ & $\leq \% 3$ \\
\hline \hline Victor 2030 & NA & 0.073 & 81.9 counts/s/nM & $\leq \% 11$ \\
\hline
\end{tabular}

TABLE 1: Performance of systems discussed.

The analytical sensitivities of the SiPM detector with cuvette, fluidic chip, and the commercial reader were calculated and are: $9.32 \cdot 10^{-2} \mathrm{~A} / \mathrm{M}, 1.82 \cdot 10^{-2} \mathrm{~A} / \mathrm{M}$ and 81.9 counts/s/nM, respectively. The performances, where available, are compared in Table 1. By taking advantage of the high sensitivity and low noise of the SiPM, low levels of ATP concentrations were measured, with good stability (variability error below 3\% during the single repetition) and reproducibility (error between repetitions around $10 \%$ at the highest repetition measured and around $20 \%$ at the lowest). Multiple analyses can be performed by designing a microfluidic chip with parallel chambers. Each chamber can have a SiPM beneath it to allow independent measurements free of interferences. The SiPMs can be multiplexed to deliver a compact system due to the reduced size of the SiPM. Further integration of the electronic interface would make it possible to create an attractive "dongle" form of instrumentation coupled to cell phones to provide the user interface, data handling and communication capabilities (Preechaburana et al., 2014). In addition, the system has a higher sensitivity than sensors proposed in literatures (Tanii et al., 2001; Young et al., 2003; Tran et al., 2007).

These results open up the possibility to fabricate and commercialise SiPM-based detection systems for bioluminescence measurements. The combination of SiPM with 3D printed microfluidic chips provides a means of creating compact, sensitive and potentially low-cost (below $2 €$ ) bioluminescence systems with wide-ranging applications in chemical and biological analysis and 
clinical diagnostics. The continuous flow analysis may allow monitoring of biological events. This feature combined with the compactness of the system may allow its positioning directly inside environmental chambers, thus facilitating sample study in real time during growth. Finally, the optimisation of the optical coupling between the reaction chamber and the detector could result in an improvement in the sensitivity of the system.

\section{Conclusion}

In this work we report the design, fabrication and testing of a miniaturised bioluminescence sensing system for highly sensitive real-time ATP detection using a 3D printed microfluidic chip coupled with a silicon photomultiplier. The use of a 3D chip reduced the consumption of reactants and solution delivery close to the SiPM increased the detection efficiency. The use of a SiPM as a detector considerably simplifies the signal analysis compared to traditional systems, providing a quantitative output signal, which does not require post-elaboration of images and/or off-line analysis.

Two configurations of the bioluminescence detection system were developed using different sample holders, i.e. glass cuvettes and 3D printed chips. These two designs delivered LoD of $3.7 \mathrm{nM}$ and 8 $\mathrm{nM}$, respectively. The round shaped bottom surface of the cuvette focused the light signal on the SiPM active area. As a result, a form of light coupling between the cuvette and the detector was obtained. On the other hand, the planar bottom surface of the 3D reaction chamber did not focus the light on the detector, resulting in a higher LoD. The comparison of the two systems clearly indicate the LoD is not only due to the SiPM sensitivity, but also to the system optical coupling.

Both systems exhibit an analytical dynamic range between $15 \mathrm{nM}$ and $1 \mu \mathrm{M}$, showing a stability error of $3 \%$, and a reproducibility error below $20 \%$, both measurements representing the worst case. We demonstrated the dynamic monitoring of ATP with a fast response time, $\sim 4 \mathrm{~s}$, and a full recover to baseline level within $17 \mathrm{~s}$.

Moreover, the bioluminescence sensing systems reported show a similar analytical dynamic range for ATP detection compared to a full-size PerkinElmer laboratory luminescence reader. In addition, the 3D chip integrated detection system may be positioned directly inside environmental chambers to monitor biological events in real time without perturbing the biological systems.

\section{Acknowledgments}

The authors would like to acknowledge Dr. P.G. Fallica, for the precious help on SiPM devices and technology, and D. Corso for the technical support. 


\section{References}

Ahn, J.M., Kim, B.C. and Gu, M.B., 2006. "Characterization of gltA:luxCDABE fusion in Escherichia coli as a toxicity biosensor", Biotechnol. Bioprocess Eng., vol. 11, pp. 516-521.

Armbruster, D.A, Tillman, M.D, Hubbs, L.M., 1994. Limit of detection (LQD)/limit of quantitation (LOQ): comparison of the empirical and the statistical methods exemplified with GC-MS assays of abused drugs. Clinical Chemistry, vol. 40, 7, pp. 1233-1238.

Available online, url: http://formlabs.com/3d-printers/form-1-plus/.

Available online, url: http://www.syringepump.com/NE-1000.php\#NE-1010.

Brolin, S. E. and Wettermark, G., 1992. Bioluminescence Analysis. VCH, New York, NY, USA.

Burri, S., Maruyama, Y., Michalet, X., Regazzoni, F., Bruschini, C., Charbon, E., 2014. "Architecture and applications of a high resolution gated SPAD image sensor”. Optics Express, vol. 22, n. 14, pp. 17573-17589.

Champiat, D., Matas, N., Monfort, B., Fraass, H., 2001. Applications of biochemiluminescence to HACCP, Luminescence, vol. 16, pp. 193-198.

Comina, G., Suska, A., Filippini, D., 2014. "Low cost lab-on-a-chip prototyping with a consumer grade 3D printer", Lab Chip, vol. 14, pp. 2978-2982.

Comina, G., Suska, A., Filippini, D., 2015. "3D printed unibody lab-on-a-chip: features survey and check-valves integration", Micromachines, vol. 6, pp. 437-451.

Comina, G., Suska, A., Filippini, D., 2015. "Autonomous chemical sensing interface for universal cell phone readout", Angewandte Chemie, vol. 54, pp. 8708-8712.

Comina, G., Suska, A., Filippini, D., 2016. "A 3D printed device for quantitative enzymatic detection using cell phones", Anal. Methods, vol. 8, pp. 6135-6142.

Comina, G., Suska, A., Filippini, D., 2016. "Towards autonomous lab-on-a-chip devices for cell phone biosensing", Biosensors and Bioelectronics, vol. 77, pp. 1153-1167.

Di Rocco, G., Gentile, A., Antonini, A., Truffa, S., Piaggio, G., Capogrossi, M.C., Toietta, G., 2012. "Analysis of biodistribution and engraftment into the liver of genetically modified mesenchymal stromal cells derived from adipose tissue". Cell Transplantation, vol. 21, n. 9, pp. 1997-2008.

Finocchiaro, P., Pappalardo, A., Cosentino, L., Belluso, M., Billotta, S., Bonanno, G., Di Mauro, S., 2009. "Features of Silicon Photo Multipliers: Precision Measurements of Noise, Cross-Talk, Afterpulsing, Detection Efficiency”. IEEE Trans. on Nuclear Science, vol. 56, n. 3, pp. 1033 - 1041.

Gracias, K.S., McKillip, J.L., 2004. " A review of conventional detection and enumeration methods for pathogenic bacteria in food". Canadian Journal of Microbiology. vol. 50, n. 11, pp. 883-890.

Libertino, S., Corso, D., Lisiansky, M., Roizin, Y., Palumbo, F., Principato, F., Pace, C., Finocchiaro, P., Lombardo, S., 2012. "Ionizing radiation effects on Non Volatile Read Only Memory cells”. IEEE Trans. Nuclear Science, vol. 59, n. 6, pp. 3016-3020.

Lundin, A., 2000. " Use of Firefly Luciferase in ATP-Related Assays of Biomass, Enzymes, and Metabolites", Methods in Enzymology, vol. 305, pp. 346-370.

Mazzillo, M., Condorelli, G., Piazza, A., Sanfilippo, D., Valvo, G., Carbone, B., Fallica, G., Billotta, S., Belluso, M., Bonanno, G., Pappalardo, A., Cosentino, L., Finocchiaro, P., 2008. 
"Single-photon avalanche photodiodes with integrated quenching resistor”, Nucl. Instrum. Meth. A, vol. 591, pp. 367-373.

Mazzillo, M., Condorelli, G., Sanfilippo, D., Valvo, G., Carbone, B., Fallica, G., Billotta, S., Belluso, M., Bonanno, G., Cosentino, L., Pappalardo, A., Finocchiaro, P., 2009. "Silicon photomultiplier technology at stmicroelectronics".IEEE Trans. Nucl. Sci., vol. 56, n. 4, pp. 2434.

Michalet, X., Ingargiola, A., Colyer, R.A., Scalia, G., Weiss, S., Maccagnani, P., Gulinatti, A., Rech, I., Ghioni, M., 2014. "Silicon Photon-Counting Avalanche Diodes for Single-Molecule Fluorescence Spectroscopy”. IEEE Journal of Selected Topics in Quantum Electronics, vol. 20, n. 6, pp. 248-267.

Min, J., 2007. "17 $\beta$-Estradiol-stimulated eNOS gene transcriptional activation is regulated through the estrogen-responsive element in eNOS promoter", Biotechnol. Bioprocess Eng., vol. 12, pp. 446449.

Molecular Probes Product Information. ATP Determination Kit (A22066), 2005.

Pagano, R., Corso, D., Lombardo, S., Libertino, S., Valvo, G., Sanfilippo, D., Russo, A., Fallica, P. G., Pappalardo, A., Finocchiaro, P., 2011. "Optimized silicon photomultipliers with optical trenches”, proc. IEEE European Solid-State Device Research, pp. 183-186.

Pagano, R., Corso, D., Lombardo, S., Valvo, G., Sanfilippo, D., Fallica, G., Libertino, S., 2012. "Dark Current in Silicon Photomultiplier Pixels: Data and Model”. IEEE Trans. Electron Devices, vol. 59, n.9.

Pagano, R., Libertino, S., Sanfilippo, D., Fallica, G., Lombardo, S., 2016. "Improvement of Sensitivity in Continuous Wave Near Infra-Red Spectroscopy Systems by using Silicon PhotoMultipliers”. Biomedical Optics Express, vol. 7, n. 4, pp. 1183-1192.

Pagano, R., Valvo, G., Sanfilippo, D., Libertino, S., Corso, D., Fallica, P.G., Lombardo, S., 2013. "Silicon Photomultiplier Device Architecture with Dark Current Improved to the Ultimate Physical Limit”. Appl. Phys. Lett., vol. 102, n. 18, pp. 183502.

Preechaburana, P., Suska, A., Filippini, D., "Biosensing with cell phones". 2014. Trends in Biotechnology, vol. 32, no. 7, pp. 351-355.

Romanova, N.A., Brovko, L.Y., Moore, L., Pometun, E., Savitsky, A.P., Ugarova, N.N., Griffiths, M.W.2003. “Assessment of Photodynamic Destruction of Escherichia coli O157:H7 and Listeria monocytogenes by Using ATP Bioluminescence”. Applied and Environmental Microbiology, Vol. 69, No. 11, pp. 6393-6398.

Santangelo, M.F., Sciuto, E.L., Busacca, A.C., Petralia, S., Conoci, S., Libertino, S., 2015. “SiPM as miniaturised optical biosensor for DNA-microarray applications,” Sens. Biosens. Res., vol. 6, pp. 95-98.

Santangelo, M.F., Sciuto, E.L., Lombardo, S.A., Busacca, A.C., Petralia, S., Conoci, S., Libertino, S., 2016. "Si Photomultipliers for Bio-Sensing Applications". IEEE J. Sel. Top. Quantum Electron.,vol. 22,n. 3.

Sciacca, E., Giudice, A.C., Sanfilippo, D., Zappa, F., Lombardo, S., Consentino, R., Di Franco, C., Ghioni, M., Fallica, G., Bonanno, G., Cova, S., Rimini, E., 2003. "Silicon Planar Technology for Single-Photon Optical Detectors”, IEEE Trans. Electron Devices, vol. 50, no. 4.

Stanley, P.E., 1989. "A review of bioluminescent ATP techniques in rapid microbiology". Journal of Bioluminescence and Chemiluminescence, vol. 4, n. 1, pp. 375-380. 
Steinberg, S.M, Poziomek, E.J., Engelmann, W.H., Rogers, K.R., 1995. "A review of environmental applications of bioluminescence measurements". Chemosphere, vol. 30, n. 11, pp. 2155-2197.

Tanii, T., Goto, T., Iida, T., Koh-Masahara, M., Ohdomari, I., 2011. "Fabrication of adenosine Triphosphate-Molecule Recognition Chip by Means of Bioluminous Enzyme Luciferase". Jpn. J. Appl. Phys., vol. 40, pp. L 1135-L 1137.

Tran, T.H., Chang, W.J., Kim, Y.B., Koo, Y.M., Kim, E.K., Yoon, J.Y., Kim, J., 2007. "The Effect of Fluidic Conditions on the Continuous-flow Bioluminescent Detection of ATP in a Microfluidic Device". Biotechnology and Bioprocess Engineering, vol. 12, pp. 470-474.

Xiong, Y.Q., Willard, J., Kadurugamuwa, J.L., Yu, J., Francis, K.P., Bayer, A.S., 2005. "Real-Time in Vivo Bioluminescent Imaging for Evaluating the Efficacy of Antibiotics in a Rat Staphylococcus aureus Endocarditis Model". Antimicrobial Agents and Chemotherapy, vol. 49, n. 1, pp. 380-387.

Young, I.T., Moerman, R., Van Den Doel, L.R., Iordanov, V., Kroon, A., Dietrich, H.R.C., Van Dedem, G.W.K., Bossche, A., Gray, B.L., Sarro, L., Verbeek, P.W., Van Vliet, L.J., 2003. “Monitoring enzymatic reactions in nanolitre wells”. Journal of Microscopy, Vol. 212, pp. 254-263. 


\title{
Supplementary Information
}

\section{Integrating printed microfluidics with silicon photomultipliers for miniaturised and highly sensitive ATP bioluminescence detection}

\author{
M.F. Santangelo ${ }^{\mathrm{a}}$, S. Libertino ${ }^{\mathrm{a}}$, A.P.F. Turner ${ }^{\mathrm{b}}$, D. Filippinic ${ }^{\mathrm{c}}$ and W.C. Mak ${ }^{\mathrm{b}}{ }^{*}$

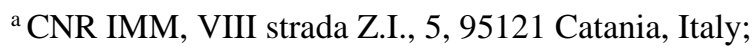 \\ ${ }^{\mathrm{b}}$ Biosensors and Bioelectronics Centre, Department of Physics, Chemistry and Biology - IFM, Linköping University, S- \\ 58183 Linköping, Sweden; \\ c Optical Devices Laboratory, Department of Physics, Chemistry and Biology - IFM, Linköping University, S-58183 \\ Linköping, Sweden; \\ *E-mail: wing.cheung.mak@liu.se; Tel.: +46-0-1328-6921.
}

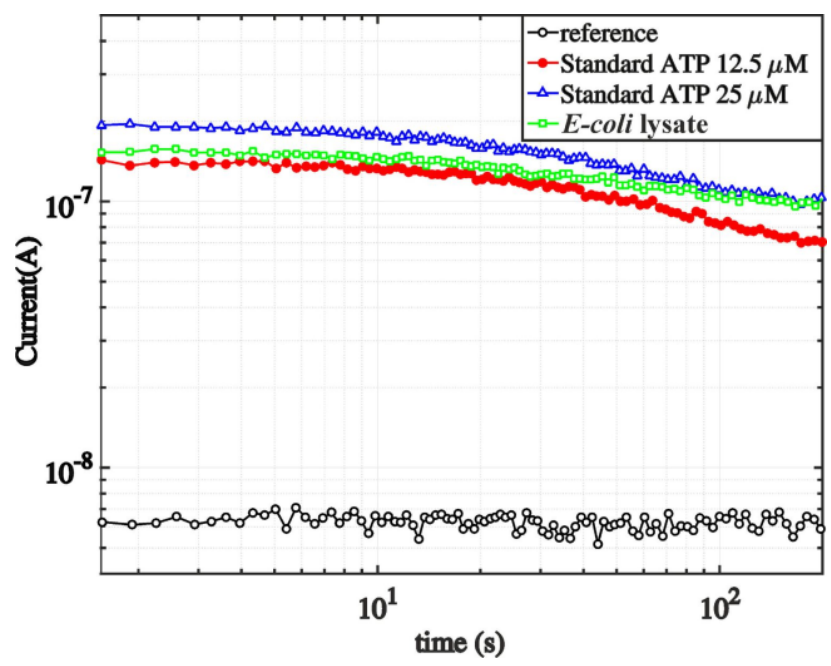

Fig. S1: Comparison of bioluminescence signals for a Standard ATP at $12.5 \mu \mathrm{M}$ (red circles), $25 \mu \mathrm{M}$ (blue triangles) and E.coli cells (green squares). The reference signal is also reported (black circles). 